\title{
Le caractère totalement inéluctable de l'histoire
}

Entretien avec le dramaturge belge Thomas Bellinck sur le rôle et l'importance de l'histoire dans son œuvre

De totale onontkoombaarheid van de geschiedenis. Gesprek met de Belgische theatermaker Thomas Bellinck over de rol en betekenis van geschiedenis in zijn werk

Traducteur : Emilie Syssau

\section{(2) OpenEdition}

Journals

Édition électronique

URL : https://journals.openedition.org/temoigner/6315

DOI : 10.4000/temoigner.6315

ISSN : 2506-6390

Cet article est une traduction de :

De totale onontkoombaarheid van de geschiedenis - URL : https://journals.openedition.org/temoigner/ 6297 [nl]

Éditeur :

Éditions du Centre d'études et de documentation Mémoire d'Auschwitz, Éditions Kimé

Édition imprimée

Date de publication : 1 octobre 2017

Pagination : $50-59$

ISBN : 978-2-930953-01-4

ISSN : 2031-4183

Référence électronique

"Le caractère totalement inéluctable de l'histoire », Témoigner. Entre histoire et mémoire [En ligne], 125 |

2017, mis en ligne le 24 décembre 2021, consulté le 03 février 2022. URL : http://

journals.openedition.org/temoigner/6315; DOI : https://doi.org/10.4000/temoigner.6315 
«L'engagement est une forme de réaction à l'histoire », disait Stéphane Hessel,

philosophe, diplomate et père du mouvement des Indignés. Un propos qui convient

bien au dramaturge belge Thomas Bellinck: I'histoire joue invariablement un rôle central dans les spectacles de la compagnie de théâtre Steigeisen qu'il a fondée avec lacteur Jeroen Van der Ven

$\rightarrow$ Jasper Delbecke (UGent)

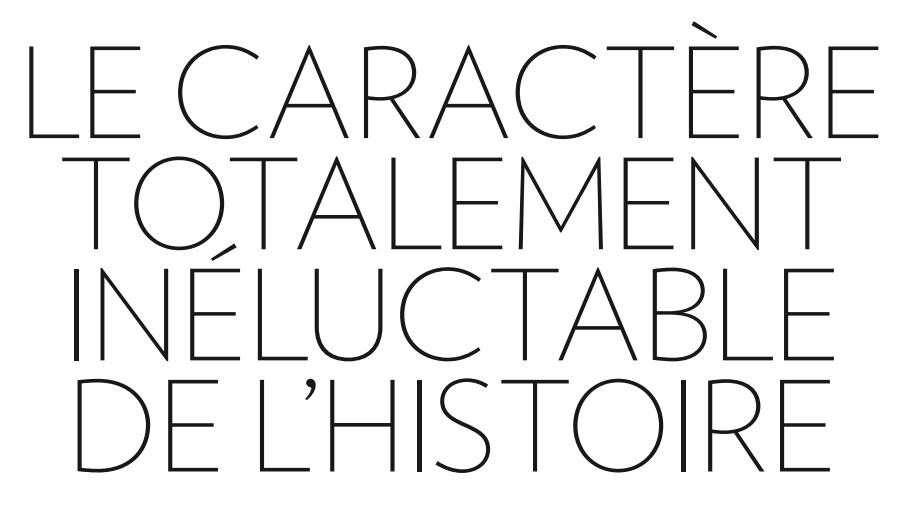

Entretien avec le dramaturge belge Thomas Bellinck sur le rôle et l'importance de l'histoire dans son œuvre

\section{Steigeisen, dans les remous de} 'histoire : de Fobbit à Memento Park

Fobbit (2009) relate l'histoire de soldats belges en Afghanistan, à l'aide de films, livres, prospectus, lettre ou encore dentretiens avec un correspondant de guerre, un cofficier de l'ape d'air envoyé 'égulierem uf officier de larmée de lair envoyé régulièrement en Afghanistan. Contraire poviviste lobecticin quécache un ês Sally, Jery and the 38 Gun (2010), Dlut aue, Billy Sally, Jerry and the .38 Gun (2010), plutot que doffrir Ford 22 seption 1975 a Sa Frasinat sur Gerald . 'hom ro Poinne. Lethal Inc. (2011) est une présentation PowerPoint exposant la recherche de [l'thodes dexécution humaines. De Onkreukelbare [L'ncoruptible] (2014) sarticule autour du personnage controversé de Maximi- auto-proclamé qui a poussé des milliers de gens sous la guillotine pendant les jours les plus houleux de la Révolution française.

Dans Memento Park (2015), vous mettez à nu les mécanismes élémentaires de l'industrie des commémorations de la "Grande Guerre ». Festivités et cérémonie commemoratives ont battu leur plein en Belgique - $e$ surtout en Flandre - a loccasion du centième anniverd'e de la Premiere Guerre mondiale. Chacun a tente den faire son beurre, tant sur le plan commercial que politiue. Des nationalistes flamands en ont profité pou raviver lheritage du souffrances inflgées aux jeunes flamands enrôlés placés sous les ordres dofficrs francophones. Avec la Tour de YYser à Diksmuide, la Flandre sest soudain imposée com ene un evernement, cest aussi décider de ne pas en commémorer un autre \#, constatait Bellinck en préambule à sa pièce (Van der Speeten 2015, p. 6). Memento Park révèl coud in in en 
- tionne le «souvenir institutionnel " imposé par les autorités. Votre texte s'appuie notamment sur des reportages dans les journaux televisés, des conferences de presse de la comedie musicale de guerre 14-18, des docuprovince de Flandre occidentale tes provinge de Flandre occidentale etles coût des commémorations.

Ce n'est pas un hasard si ce spectacle joue avec le re-enactment (reconstitution) historique, une pratique largement répandue. Des historiens amateurs ou professionnels font revive le passé en rejouant des batailles et en restituant des villages médievaux. Frederik Le Roy désigne lexpocations theátrales du passépar lexpression "theâtre taxidermique»; ce sont pour lui des "formes théatrales "ésireuses de faire "vivre" un passe "mort" a laide d"une représenp. 13) L" p. 13) Létude de ces mécanismes Selon Frederik Le Roy, la mise en scène réaliste de lhistoire que vous

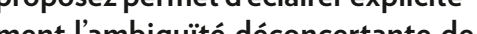
ment lambiguité déconcertante de la à fois u présent ou a passé est à la fois "présent "et "passé ", "thétrale " et "authentique ", 25). Ce n'est pas un asal p. 25). Cen'est pas un hasard si lon de da jouvent qualifie Steigeisen de compagnie de theâtre au cœur desremous delhistorie; cette description ne couvre cependant plus tout à fait la portée de en 2016

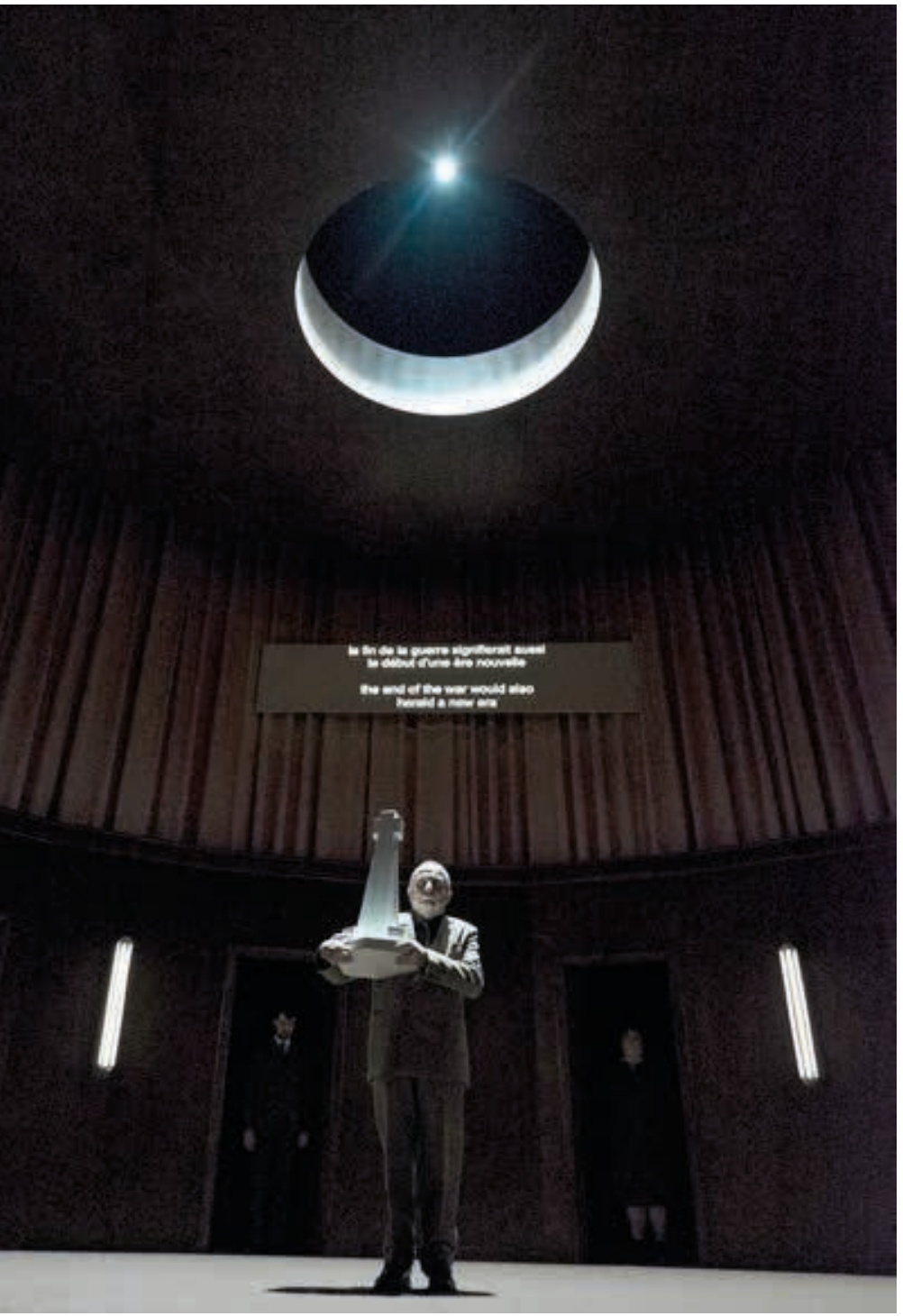

'enjeu du spectacle consistait donc aussi à tenter de montrer ce processus : que se passe-t-il si on pren de montrer ce processus : que se passe-t-il si on prend tion du déroù lementhistorique dectter d'une réflexion théâtrale. Contre culles lis en dune rexion quels révolutionnaires? Nous volions établir tos palles évén no

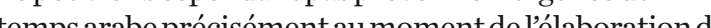

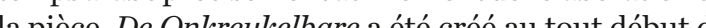
cette révolution Ce cetcen Afrique du Nord avait complètement dérallé, que De Onkreukelbar avait conplètement deraille, que De On

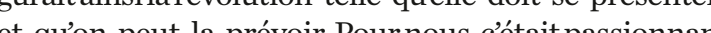
de contar que l'on fit don

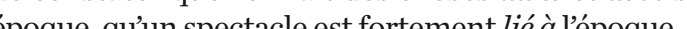

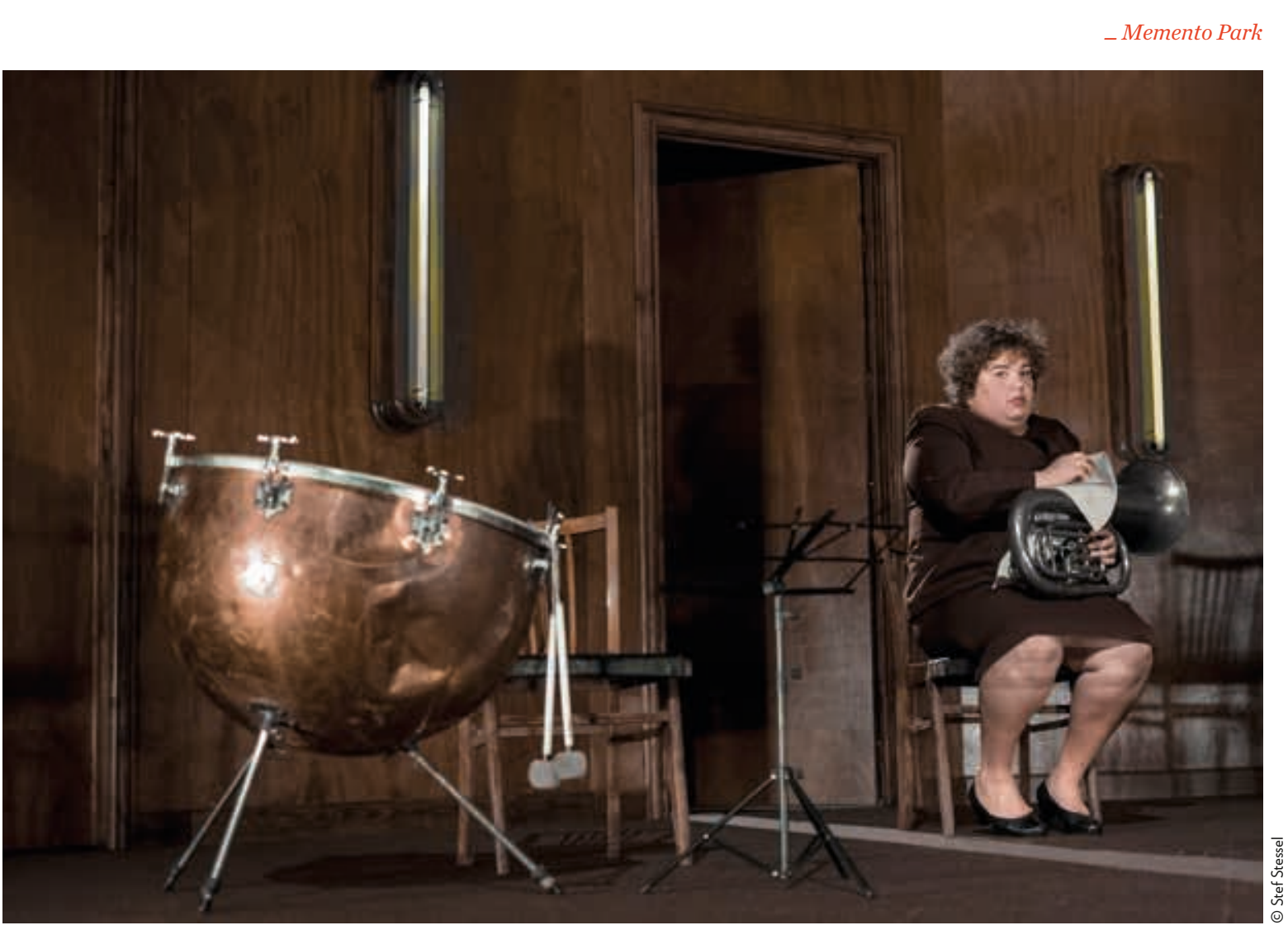
L'histoire, une responsabilité partagée :
Het Mirakel van Almería

La zone de tension entre petite et grande Histoire, La zone de nelle souvenir indide 'Alme t lá re un dernien

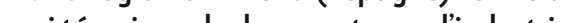
lican

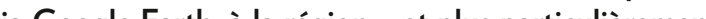

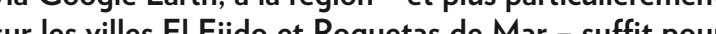
remar vill

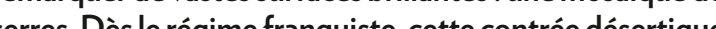
sertique a été conquise pour raisons économiques. Des système ' 'roduit a joud'hu (nom

Thomas Bellinck : Cette description vaut toujours pour Billy, Sally, Jerry and the .38 Gun et pour Lethal lnc. Dans ces spectacles, nous utilisons vraiment Onkreukelbare, nous prono Onkreukelbare, nous prenons davantage de distance, sont pour nous un prétexte pour parler des procespar partir en vrille. La pièce s'appuie sur l'ouvrage $O n$ Révolution françise a été un brouillon destule que la révolution françase a eté un brouilon de la Revolution russe. Cette analyse ma intrigué en avançantl'idée tous les élém pourrait parler d'une sorte de re-enactment. 
-. Après la chute de Franco, des immigrés clandestins essentiellement, ont afflué pour effectuer ce rude traval dans la chaleur torride. Het Mirakel van Almería montre impact considérable sur l'homme et l'environnement.

Bellinck: Het Mirakel van Almería comprend globalement trois parties : passé, présent et futur. Pour chacune, nous avons voulu aborder un groupe de population spécifique pour bâtir l'histoire d'Almería. mémoration institutionnelle, nous nour de la com-

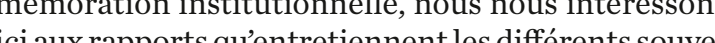
Me

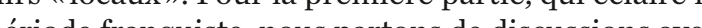
période fran des personnes âgées. Nous avons été frappés par la Almerí Nous avons donc décidé d'effectue Tranco à dería. Nousa go cille voture, avons cherché nosie de Fraé une vieille voiture, avons cherché un sosie de Franco et Tre objectif imméch otre objeća inte, mais de convoquer prosos viseres vivaces et de déclencher des réactions. Parmi les persones âges se trouvient aussibin des franquistes notoires que des personnes dont de proches parents ve retrouvaient tous dans un coin de désert à disco du costume d'un acteur jouant Franco.

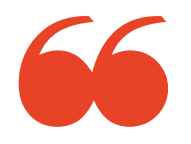

Il ne suffit pas de faire des discours forts et d'adopter des points de vue controversés.

Les dramaturges doivent aussi réfléchir à leur propre position. Ils ne peuvent pas faire fi de la complexité dont ils sont souvent eux-mêmes partie prenante.

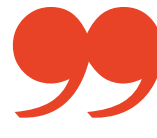

Ce choix de reconstituer la visite de Franco fait fortement penser à la démarche du réalisateur et documentariste Joshua Oppenheimer dans The Act of Killing (2012). Dans ce documentaire récompensé par plusieurs prix, Oppenheimer demande à des membres d'anciens escadrons de la mort en Ind

Bellinck: Ce que je trouve problématique dans The Act of Killing, et que nous avons essayé d'éviter avec Het Mirakel van que nous avons essaye deviter avec d'u Mirakel van Almeria, ce sont les efforts en faveur récit vers une prise de concior fart progresser son mét vers 'nempare d'eux quand ils vivent à leurs dépen malaise senpare deux quandils viventa leurs dépen leurs propres crimes, mais jy vois surtout le résulta du montage manipulateur d Oppenheimer. Les crimippose suppose que ça a èté filmé à difénts moments. Le moments.

Pour ces raisons, je trouve le film moralisateur nous ne voulions pas tomber dans ce piège avec Het Mirakel van Almería. En outre, contrairement à la position extérieure d'Oppenheimer en Indonésie, en tant qu'Européens, nous n’avons pas la conscience vraisuille concernant la situation à Almería. Jachète vésures légumes venant des serres dilmerla. Moon Blaisse et moi sommes a la fois réalisateurs du film et consommateurs, et à ce titre partie prenante dans cette histoire. Notre rapport à notre sujet est différent de celui d'Oppenheimer au sien. Nous ne cherchons pas a provoquer une prise de conscience, mais à représenter avec tous les acteurs du recit une trame dont nous, realisateurs, faisons (tout comme les spectateurs) indissociable-
ment partie.

Le parallèle avec The Act of Killing se retrouve surtout dansle premierépisode de Het Mirakel van Almería (concernant le passé). À l'instar d'Oppenheimer, nous avons délibérément opté pour une perspective unilatérale, a savoir celle des premiers pionniers venus dans cette région à l'époque de Franco. Les «exploiteurs » daujourd hui sontles migrants d'hier, mais ils'ont visiblement un peu oublié. Le deuxième volet («présent») évoque les frictions entre ces pionniers et les nouveaux la région. Dans le dernier volet, nous posons des ques- tions sur l'avenir d'Almería. Nous avons tenu avec tous les acteurs un congrès pour et sur la région, visiblement sans avenir Les sources naturelles sontépuisées par des années de culture intensive, tout comme l'ea douce, d'où le recours à des stations d'épuration onéreuses, et la progressive délocalisation de la culture de fruits et légumes en Afrique du Sud. C'est précisémen Killing : noi, la principale diftere avec The Act of exportateurs, des ins tant exportateurs, des innigrants clandestins, des directeurs de banque te des consonmateurs. La réalite est à Complexe qu' il nous est in une position mairement Mirakel van Almería ne parle pas du bien et du mal mais de la viole mais de la violence du systène qui fait que chacun est lités partagées.

À lépoque de De Onkreukelbare, votre travail et cel de Steigeisen était truffé de "surprise », de "passion et de "révolution ». Vous n'empruntez plus cette voie activiste. La colère est toujours là, mais vous la canalsez à présent autrement. Car selon vous, des prises de position politiques aud théâtre captivant.

Bellinck: Il ne suffit pas de faire des discours forts et d'adopter des points de vue controversés. Les dramaturges doivent aussi réfléchir à leur propre position Ils ne peuvent pas faire fi de la complexité dont ils sont souvent eux-mêmes partie prenante. Mon activisme souvent eux-mêmes partie prenante. Mon activisme
s'exprime aujourd'hui dans des spectacles et des projets qui soulignent cette ambiguïté. On ne combat pas le populisme croissant en dénonçant des coupables, mais en endossant la responsabilité d'un passé commun On ne fait que porter la culpabilité, alors que l'on peut endosser la responsabilité, c'est quelque chose d'actif endosser la respons.

Pour moi, c'est là la définition de l'activisme: crée des narrations, peu importe la forme, qui dynamisent et impliquent pour l'avenir. Nous avons échoué dan notre échec, à petite et à grande échelle. Et chaque fois, éche à petite et à grande échelle. Et chaque bilité ou de celle d'autrui. Je de notre propre culpad'autres pistes à explorer ; seule quil y a quantite d'autres pistes a explorer; seulenent, on manque aujourd'hui d'imagination pour les relever et leur

\section{À propos de la nostalgie et de l'histoire,} hypothèque sur l'avenir:

"Le passé est plus vendeur que le futur " constatait Andreas Huyssen dans Present Pasts. Urban Palimpsests Andes Huyssen dans Present Pasts. Urban Palimpsest and the Politics of Memory (2003). Un nuage toxique socié sccictecidente lisation a comme une arme dans un monde oú la mondialisation a atteít ses extrémités et où les perdants exprjelime leur mécontentement. Svetlana Boym le résume is nostgic not for hepast hewayitwa but for he is nostalgic not for he past he wayit was, but for the past . passé tel qu il était, mais du passé tel qu il aurait pu être (Boym, 2001, p. 351). Dans Domo de Etropa Historio en ( péenne en exil), vous jouez sur ces sentiments. Il s'agit d'u musée fictif construit tout près du quartier europeen de Bruxelles. Inscrit dans un futur proche, ce musée revien Iurf histoire de Inion europeenne, dont vous imaginez l'un press-citron port Mabios, d'un prosse-citron portant la tête dAngela Merkela une collection de réglementations européennes ute de l'Union européenne.

Bellinck : J'utilise la nostalgie comme un ressor héâtral : c'est tout à la fois un instrument et une critique de son emploi par les musées. Le Parlamentarium de Bruxelles en est un bel exemple. Ce centre de l'unification européenne et explique le fonctioncor cúc En termes de dramaturgie architecturale, le Paen. En termes de dramaturgie architecturale, le Parlamentarium présente de nombreuses similarités avec tilisentla natrie pour moteur, etconmen urration narration par un cataclysme gigantesque : la Seconde Guerre mondiale pour le Parlamentarium, et l'exploipour le musée du Parti communiste. Par ailleurs, le pourcours des deux musées commence en sous-sol. visiteur em deux nusees conmente en sous-sol. Le visiteur emprunte un long couloir sombre et ne voit pae des images de douleur, de destruction et datrocités dans un espace très éclairé - bleu au Parlamentarium, . 
« Cela reste une loi inéluctable de l'histoire : elle défend précisément aux contemporains de reconnaître dess leurs commencements les grands mouvements qui déterminent leur époque.» Stefan Zweig (1881-1942) sut ansi conter a la ve de la Sendiale. Dans Die Wet von Gestern [Le Monde d hier], I'ecrivain autrichien d ora XIXe diècle. Près de 70 ane plus tordre mondial né au XIX siècle. Près de 70 ans plus tard, nous croyon revivre une époque semblable. Lordre mondial qui cst aujou l'hu rodujé Populiste do nières années est aujourd hui redessiné. Populisme, nationalisme et conservatisme progressent, et immanquablement surgit (nscrire à cette idée.

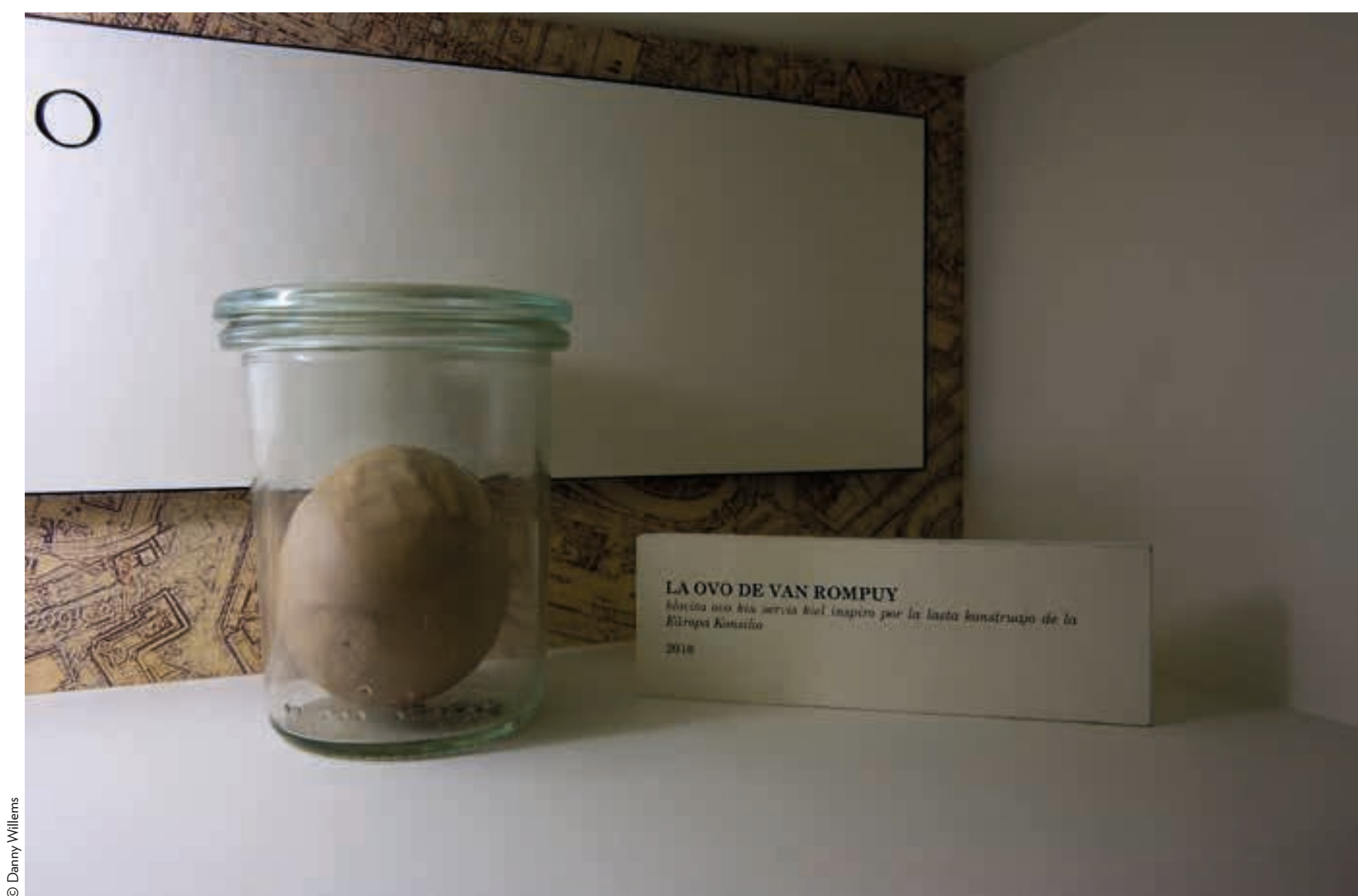

Bellinck: C'est la maladie de notre époque de revenir aux périodes historiques antérieures pour expliquer les ances contemporaines. Selon moi, cette façon de faire, employée autant par les gens de gauche que par ceux de droite, apporte peu au débat. Je trouve que c’est un réflexe dangereux. Mais si je devais comparer notr époque à une période antérieure, ce ne serait sûrement pas avec les an XIXe 1930, mais avec la seconde moite une for du XIXe sibcle - une epoque marqué par lisetion mond on de l'économi qu ence la nénon d'éta la polictique. On ámsistait alors à la naissance dêtats-nations é à lénergence dune de 1873 'os a de perceptible dans le monde entier. Au nême moment pogra pogroms russes. Je suis dóne tenté die dire que nous daus trouvons dans une période silnilaire, plutôt que dans les années trente. Jail le sentiment que survient encen l'échiquier Nos pouv, andéplacer toutes les directions.

Exilé au Brésil,Stefan Zweigs'est suicidépeu avan que n'éclate la Seconde Guerre mondiale, de désarroi face à l'égarement de «son» Europe. La mort de l'écrivain prouve selon moi le caractère totalement inéluctable de l'histoire. Je sais ce que c'est pour un cosmopolite et un intellectuel que de ne plus se sentir chez soi dans les remous actuels du temps. Mais je crois que je suis plus optimiste que Zweig. Plutôt un pessimiste constructiviste. (rires)

Traduit du néerlandais par Emilie Syssau

Pour en savoir plus

$\diamond$ Vous pouvez consulter le numéro 118 de Témoigner. Entre histoire et mémoire (septembre 2014) : entretien avec Joshua
Oppenheimer à propos de son documentaire The Act of Killing et de la suite. The Look of Silence.
Bibliographie

$\diamond$ Boym, Svetlana (2001): The Future of Nostalgia, New York Basic Books.

$\rightarrow$ Chamayou, Grégoire, (2010): Les chasses à 'thomme : histoire et philosophie du pouvoir cynégétique, Paris, La Fabrique.

$\triangle$ Coussens, Evelyne (2012) : “"Wij worden voortdurend ingehaald door de tijd" Steigeisen zet zich schrap in de maalstroom van de geschiedenis ", Etcetera: Tijdschrift voor podiumkunsten 130, p. 22-28.

$\diamond$ Delbecke, Jasper (2015) : «"Zou een heel klein beetje oorlog soms niet beter kunnen zijn": over de WO I herdenkingen, Thomas Bellinck / Steigeisens Memento Park en Eventization n,

$\diamond$ T.J. Demos, T.J. (2013) : The Migrant Image: The Art and Politics of Documentary during Global Crisis, Durham \& London

$\diamond$ Fischer-Lichte, Erika (2002) : History of European Drama and Theatre, traduit de l'allemand par Jo Riley London \& New York, Routledge.

$\triangle$ Fukuyama, Francis (1992): End of History and the Last Man, ree Press, traduit de l'anglais par Denis-Armand Canal sous I $\diamond$ Hartog, François (2003): Régimes d'historicité. Présence et expériences du temps, Paris, Seuil. $\diamond$ Huyssen, Andreas (2003): Present Pasts. Urban Palimpsest
and the Polititics of Memory, Stanford, Stanford University Press. $\diamond$ Le Roy, Frederik (2012) : Verknoopte tijd, verfrommelde geschiedenis. een theaterwetenschappelijk en geschiedfilosofisch onderzoek naar theater en performance als politiek van de herinnering in het modern en presentistisch historiciteitsregime,
thèse de doctorat, Université de Gand, Faculté de philosophie et lettres \& Wijsbegeerte.

$\diamond---$, « Realistische rituelen. De documentaire dubbels van Mil Rau en het International Institute of Political Murder ", Etcetera

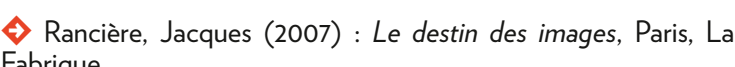

$\diamond$ Van der Speeten, Geert : “ "Ik wil alles op zijn kop kunnen
zetten": Thomas Bellinck maakt "Memento Park" voor de KVS ", zetten": Thomas Bellinck maakt “Mem
De Standaard, 31 januari 2015, C6-C7. $\triangle$ Weber, Samuel (2004): Theatricality as Medium, New York,
Fordham University Press. 